\title{
Role of Dipyrone in the High On-Treatment Platelet Reactivity amongst Acetylsalicylic Acid-Treated Patients Undergoing Peripheral Artery Revascularisation
}

\author{
Jan Hartinger ${ }^{a}$ Robert Novotny ${ }^{b}$ Jana Bilkova ${ }^{d}$ Tomas Kvasnicka ${ }^{d}$ \\ Petr Mitas ${ }^{c}$ Martin Sima ${ }^{a} \quad J a r o s l a v ~ H l u b o c k y{ }^{c} \quad$ Jan Kvasnickad ${ }^{d}$ Ondrej Slanara \\ Jaroslav Lindnerc \\ a Department of Clinical Pharmacology and Pharmacy, Institute of Pharmacology, General University Hospital and

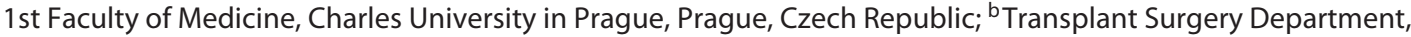 \\ Institute for Clinical and Experimental Medicine, Prague, Czech Republic; ${ }^{\text {}}$ 2nd Department of Cardiovascular \\ Surgery, General University Hospital and 1st Faculty of Medicine, Charles University in Prague, Prague, Czech \\ Republic; ${ }^{\mathrm{d}}$ Thrombotic Centre, General University Hospital, Prague, Czech Republic
}

\section{Significance of the Study}

- This study evaluated the effects of dipyrone (metamizole) on the effectiveness of acetylsalicylic acid in patients with peripheral arterial disease after surgical revascularisation on the lower extremities. It demonstrates that in patients with peripheral arterial disease, dipyrone can induce resistance to acetylsalicylic acid. This is important as many vascular centres use dipyrone as a postoperative analgesic.

\section{Keywords}

High on-treatment platelet reactivity - Vascular surgery .

Peripheral arterial disease - Dipyrone

\begin{abstract}
Objective: To evaluate the effects of dipyrone on sensitivity to aspirin (acetylsalicylic acid [ASA]) in patients who underwent peripheral artery vascular reconstruction. Subjects and Methods: Impedance aggregometry and light transmission aggregometry were used to determine the effects of dipyrone on ASA treatment in 21 patients. Blood samples were drawn in a 7-day period after the surgery. The cut-off value for high on-treatment platelet reactivity (HTPR) was set at $<$
\end{abstract}

$65 \%$ of aggregation inhibition for impedance aggregometry. For light transmission aggregometry the cut-off value for arachidonic acid-induced aggregation was set at $>20 \%$ of aggregating platelets, and the cut-off value for epinephrineinduced aggregation was $>44 \%$ of aggregating platelets. The cut-off value for each method was derived from a large number of patients treated with a daily dose of $100 \mathrm{mg}$ of ASA. Results: We found HTPR in 14 (67\%) of the 21 patients. None had primary resistance to ASA, i.e., after the addition of ASA in vitro all samples showed antiplatelet efficacy. Regression analysis showed a possible correlation between lower efficacy of ASA treatment and higher daily doses of dipyrone ( $p=0.005$ for impedance aggregometry, $p=0.04$ for light transmission aggregometry), higher platelet count

\begin{tabular}{ll}
\hline KARGER & $\begin{array}{l}\text { (c) } 2018 \text { The Author(s) } \\
\text { Published by S. Karger AG, Basel }\end{array}$ \\
E-Mail karger@karger.com & $\begin{array}{l}\text { This is an Open Access article licensed under the Creative Commons } \\
\text { Attribution-NonCommercial-4.0 International License (CC BY-NC) } \\
\text { (http://www.karger.com/Services/OpenAccessLicense), applicable to } \\
\text { the online version of the article only. Usage and distribution for com- } \\
\text { mercial purposes requires written permission. }\end{array}$
\end{tabular}

Robert Novotny

Transplant Surgery Department, Institute for Clinical and Experimental Medicine Videnska 1958/9

CZ-140 21 Prague 4 (Czech Republic)

E-Mail novotny_robert@hotmail.com 
( $p=0.005$ for impedance aggregometry), and shorter time from surgery ( $p=0.03$ for impedance aggregometry). Conclusion: HTPR occurs in $67 \%$ of ASA-treated patients after lower limb vascular surgery. The occurrence of HTPR correlates with the daily dose of dipyrone. Therefore, dipyrone should not be used as a postoperative analgesic in ASAtreated patients after peripheral artery revascularisation due to its influence on the effectiveness of ASA.

(C) 2018 The Author(s)

Published by S. Karger AG, Basel

\section{Introduction}

Low-dose aspirin (acetylsalicylic acid [ASA]) therapy reduces by $40 \%$ the incidence of coronary and peripheral artery closures after surgical revascularisation [1]. Despite the high efficacy of aspirin in the prevention of secondary cardiovascular events, up to $27-28 \%$ of patients do not respond to aspirin antiplatelet therapy. This high on-treatment platelet reactivity (HTPR) is associated with significantly higher risks of developing acute coronary syndrome, vascular graft failure, cerebrovascular events, and higher mortality $[2,3]$.

Even though HTPR is not strictly defined and its definition varies between different studies, high platelet reactivity despite ASA treatment has been repeatedly described as a negative prognostic factor for the further clinical course [3-7]. A meta-analysis by Krasopoulos et al. [2] showed an odds ratio of 4.06 for acute coronary syndrome, 3.78 for stroke, and 5.99 for overall mortality in HTPR patients; the odds ratio for any cardiovascular event is $3.8[2,7]$. Patients diagnosed with HTPR early after coronary artery bypass grafting had the worst prognosis in the year following the surgery $[8,9]$, and the perioperative HTPR also correlated with venous coronary bypass obliteration [6].

Only one study [5] examined the incidence and clinical significance of aspirin resistance in patients with peripheral arterial disease (PAD). After balloon angioplasty of lower extremity arteries, a correlation between aspirin resistance and throughput-affected arteries was confirmed during annual monitoring. ASA resistance was defined as a lack of inhibition of platelet aggregation upon induction with collagen (COL) and adenosine diphosphate (ADP) measured by impedance aggregometry. In a group of 100 patients studied, there were 8 occlusions of arteries where laboratory findings confirmed ASA resistance. The patients were checked 4 times during a year. During each measurement performed on these patients, changes in the sensitivity to antiplatelet therapy were no- ticed in terms of both increases and decreases in ASA effectiveness.

Dipyrone (metamizole) and other non-steroidal antiinflammatory drugs (NSAIDs) have been shown to interfere with the efficacy of ASA by inducing HTPR [10-14]. The objective of this study was to evaluate the incidence of HTPR as well as the effect of dipyrone on antiplatelet treatment in patients who underwent peripheral artery revascularisation.

\section{Subjects and Methods}

\section{Patient Selection}

A total of 21 patients (5 women, 16 men) with PAD who had undergone vascular procedures with no complications were selected for our study. The inclusion criterion was a daily dose of 100 $\mathrm{mg}$ of aspirin at least 6 days before testing for platelet reactivity. The exclusion criterion was a hospital stay $<6$ days. The average age of the patients was $68.9 \pm 4.8$ years. All patients were tested within the first 30 days after revascularisation of a lower extremity. Fifteen patients had undergone a bypass procedure (prosthetic, venous, or allograft bypass) and 6 patients had undergone other types of surgery/intervention (patch, thrombectomy, transmetatarsal amputation, percutaneous transluminal angioplasty, or angiography).

\section{Investigation of Platelet Activity}

Two different methods were used for the evaluation of platelet function: light transmission aggregometry (LTA) (PAP 8E Analyzer; Bio/Data Corporation, USA) and impedance aggregometry (Multiplate Analyzer; Roche, Munich, Germany) [15, 16]. For LTA, arachidonic acid (ARA), epinephrine (EPI), ADP, and COL were used as aggregation inducers.

COL was not used for HTPR determination because of its lower specificity for inhibition of aggregation induced by ASA when compared to ARA or EPI. ADP results were not used because the results of ADP-induced aggregation are influenced by concomitant clopidogrel use, which was common in our patients. Based on results obtained from a large group of ASA-treated patients, the cut-off value for HTPR was set at $<65 \%$ platelet aggregation inhibition in the impedance aggregometry tests. For LTA, the cut-off value for ARA-induced aggregation was set at $>20 \%$ of aggregating platelets, and the cut-off value for EPI-induced aggregation was set at $>44 \%$ of aggregating platelets.

Impedance aggregometry was performed using whole blood. The TRAP assay with the inducer TRAP 6 was used for checking the total reactivity of platelets. TRAP is an activating peptide for thrombin receptors. This test is independent of antiplatelet therapy with ASA. Two tests were used to monitor the effectiveness of ASA: the ASPI test and the ASPI ASA test. The inducer in these tests is ARA (Roche; final concentration $0.5 \mathrm{mM}$ ). In this assay, the addition of $30 \mathrm{mg} / \mathrm{mL}$ ASA in vitro was used as a control test for the detection of primary resistance to ASA. The results are expressed as percent efficacy of ASA. For clinical use, efficiency is determined using a cut-off value of average efficiency at a daily dose of $100 \mathrm{mg}$ of ASA. Patients identified as sensitive to aspirin therapy have a $65-100 \%$ effectiveness of therapy. 
Table 1. Patient medical history and characteristics $(n=21)$

\begin{tabular}{llrl}
\hline Subgroup & $\begin{array}{l}\text { ASA sensitive } \\
(n=7,33 \%)\end{array}$ & $\begin{array}{l}\text { HTPR } \\
(n=14,67 \%)\end{array}$ & $p$ \\
\hline Female & $1(14)$ & $5(36)$ & 0.608 \\
Bypass surgery & $3(43)$ & $12(86)$ & 0.124 \\
Other revascularisation (PTA, thrombectomy, patch) & $4(57)$ & $2(14)$ & 0.124 \\
Active smoking & $2(29)$ & $5(36)$ & 1.000 \\
Diabetes mellitus & $1(14)$ & $3(21)$ & 1.000 \\
Arterial hypertension & $4(57)$ & $11(79)$ & 0.608 \\
Chronic kidney disease $^{1}$ & $1(14)$ & $3(21)$ & 1.000 \\
Coronary artery disease $_{\text {Carotid artery disease }^{2}}$ & $2(29)$ & $6(43)$ & 0.874 \\
\hline
\end{tabular}

ASA, acetylsalicylic acid; HTPR, high on-treatment platelet reactivity; PTA, percutaneous transluminal angioplasty. ${ }^{1}$ Including 1 patient in the chronic haemodialysis programme. ${ }^{2}$ Including 1 patient after ischaemic stroke.

Table 2. Pharmacotherapeutic information $(n=21)$

\begin{tabular}{llll}
\hline Subgroup & $\begin{array}{l}\text { ASA sensitive } \\
(n=7,33 \%)\end{array}$ & $\begin{array}{l}\text { HTPR } \\
(n=14,67 \%)\end{array}$ & $p$ \\
\hline Concomitant clopidogrel & $5(71)$ & $7(50)$ & 0.640 \\
Clopidogrel resistant $^{1}$ & $3(60)$ & $2(28)$ & 0.621 \\
Concomitant anticoagulation $_{\text {Concomitant dipyrone }}$ & $5(71)$ & $12(85)$ & 0.844 \\
Acetaminophen & $5(71)$ & $14(100)$ & 0.189 \\
Opioids (dihydrocodeine, tramadol, oxycodone) & $1(14)$ & $5(36)$ & 0.608 \\
Proton pump inhibitors & $0(0)$ & $5(36)$ & 0.204 \\
HMG-CoA reductase inhibitors (statins) & $3(43)$ & $5(36)$ & 1.000 \\
Allopurinol & $1(14)$ & $2(14)$ & 1.000 \\
Calcium channel blockers & $2(29)$ & $1(7)$ & 0.508 \\
ACE inhibitors & $0(0)$ & $7(50)$ & 0.072 \\
ARBs (sartans) & $2(29)$ & $5(36)$ & 1.000 \\
Diuretics (e.g., indapamide, hydrochlorothiazide, & $0(0)$ & $3(21)$ & 0.508 \\
$\quad$ furosemide, spironolactone, amiloride, and & & & 0.874 \\
$\quad$ combinations) & $2(29)$ & $6(43)$ & 1.000 \\
\hline -Blockers & $3(43)$ & $7(50)$ & \\
\hline
\end{tabular}

ASA, acetylsalicylic acid; HTPR, high on-treatment platelet reactivity. ${ }^{1}$ Clopidogrel users only.

If tested samples showed discrepant results, an additional platelet function test on a PFA-200 (Siemens, Germany) or VerifyNow (Accumetrics, San Diego, CA, USA) was performed in order to determine platelet function. Correlations between continuous variables and the percentage of platelet inhibition measured by LTA with ARA as an inducer, LTA with EPI as an inducer, and impedance aggregometry were examined. The impact of postoperative HTPR on individual clinical outcomes was not monitored.

Role of Dipyrone in Acetylsalicylic Acid Resistance

\section{Statistical Analysis}

All variables (listed in Tables 1 and 2) were tested for normality via tests on skewness and kurtosis, as well as by KolmogorovSmirnov and Shapiro-Wilk tests. To verify statistically significant correlations between variables pairwise, the Pearson correlation coefficient was used; $\chi^{2}$ analysis was used with Yates's correction for categorical variables. The Student $t$ test was used for comparison of platelet counts between ASA non-responders and ASA responders. Linear regression analysis was used for continuous variables. For visualisation of the results, GraphPad Prism 3.02 (GraphPad Software, Inc., La Jolla, CA, USA) was used. 


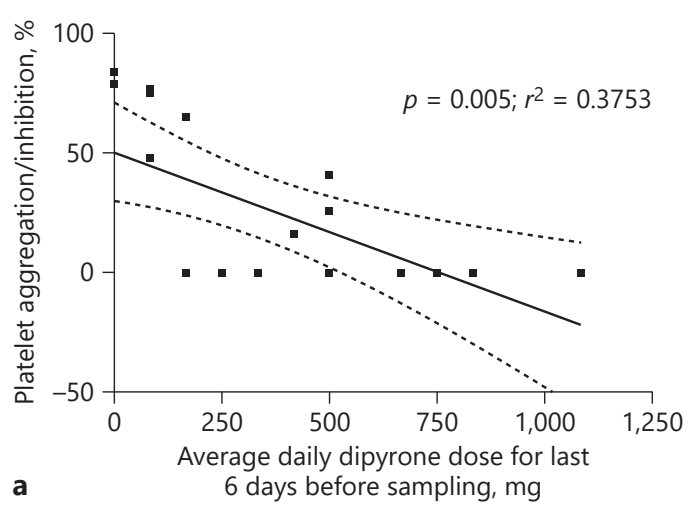

\section{Results}

Detailed patient characteristics are summarised in Table 1. More patients had HTPR despite ASA treatment after vascular bypass grafting than after other procedures ( 80 vs. $33 \%, p=0.1$ ). Gender, smoking habits, and concomitant diseases (diabetes mellitus, arterial hypertension, chronic kidney disease, coronary artery disease, and carotid artery disease) were equally distributed among HTPR patients and patients with effective antiplatelet ASA treatment (Table 1). The use of clopidogrel, anticoagulants, proton pump inhibitors, statins, allopurinol, calcium channel blockers, ACE inhibitors, ARBs, diuretics, and $\beta$-blockers was not significantly different between HTPR patients and patients with effective antiplatelet treatment (Table 2).

Platelet counts were examined in 16 of the 21 patients. Only 6 patients, with known platelet counts at the time when blood samples were drawn, had an adequate response to ASA treatment. These patients had significantly lower platelet counts than the HTPR patients (274.8 \pm 31.9 vs. $436.5 \pm 40.7, p=0.01$ ).

With the use of linear regression analysis, age, weight, and BMI did not significantly influence ASA sensitivity expressed as the percentage of aggregating platelets detected by impedance aggregometry and LTA. A significant correlation between platelet counts and the results of impedance aggregometry was found $(p=0.005)$, while LTA showed no such relationship. The longer the period after surgery, the higher was the efficacy of ASA treatment as measured by impedance aggregometry $(p=0.03)$. The higher the average dipyrone daily dose, the lower was the ASA effectiveness as measured by impedance aggregometry $(p=0.005)$ and LTA when EPI was used as an inducer $(p=0.04)$. The correlation between LTA results and an average daily dose of dipyrone was not significant when ARA was used as an inducer (Fig. 1). The results of impedance aggregometry correlated with the LTA results when ARA $(p=0.001)$ or EPI $(p=0.04)$ was used as an inducer. The pharmacotherapeutic details are summarised in Table 2.

No association was found between the tested variables and LTA results when COL was used as an inducer. In all, $57 \%$ of the patients were treated with dual antiplatelet therapy. ADP was also tested as an inducer in LTA tests. The results did not significantly correlate with daily dipyrone dose, time from surgery, and platelet counts in the whole patient group as well as in the patients treated with dual antiplatelet therapy [16] or aspirin alone [17]. The correlation missed statistical significance for platelet 
count $(p=0.070)$ and time from surgery $(p=0.079)$ in the patients not treated with clopidogrel. Nevertheless, the number of patients tested was too small to detect a significant difference between clopidogrel users and nonusers in LTA ADP tests $(p=0.069)$. The ASPI results in multiple tests did not differ significantly between the patients treated with dual antiplatelet therapy and those treated with aspirin alone $(p=0.314)$.

\section{Discussion}

Our study demonstrates that HTPR occurs despite treatment with ASA in two-thirds of patients with PAD. This high number of HTPR patients is probably caused by recent surgical intervention, which is known to be a significant contributor to the development of HTPR in surgical patients. A possible reason for transient postsurgical HTPR is a higher platelet turnover in this clinical setting or higher COX-2 expression in platelets after surgical interventions [18-21]. Although several reports have described resistance to antiplatelet therapy in patients undergoing a percutaneous intervention or coronary arterial bypass grafting or after stroke, limited data describing resistance to aspirin amongst patients with PAD are available [2, 5, 17-19, 22-24]. We have found only one study which examined the incidence and clinical significance of HTPR in patients with PAD [5].

Regression analysis showed a negative effect of a higher platelet count and a higher average daily dose of dipyrone on platelet reactivity in ASA-treated patients detected by impedance aggregometry. The negative correlation of ASA efficacy with average daily dipyrone dose was also significant in LTA testing if EPI was used as the inducer of platelet aggregation. No such correlation was observed when ARA was used as the inducer of platelet aggregation in LTA testing. This suggests that the difference between impedance aggregometry and LTA shows different predictive values for various platelet function tests $[25,26]$. Several reports have shown that analgesic therapy with NSAIDs have a significant effect on the efficacy of aspirin [10-14]. The significance of this interaction was proven in healthy volunteers for ibuprofen and dipyrone, which are widely used as perioperative analgesics in Central Europe [10]. Also, a few observational studies have confirmed the clinical importance of adverse interactions between ASA and NSAIDs in clinical settings [12, 13, 27]. In patients with coronary artery disease, dipyrone was shown to nullify the antiplatelet efficacy of ASA soon af- ter coronary artery bypass graft surgery [14]. This effect can be overcome by administering aspirin before dipyrone [28]. We have shown that medication with dipyrone should be weighed carefully against the risk of a lower effectiveness of ASA therapy in patients after lower extremity vascular surgery. We did not study the effect of other NSAIDs used in different clinical settings $[29,30]$, since only dipyrone, acetaminophen, and opioids are used in our department.

All patients receiving angiotensin receptor blockers, calcium channel blockers, and opioids had HTPR, but this was most likely due to the small number of patients using these medications. Concomitant clopidogrel therapy did not significantly affect the results of the impedance aggregometry tests. The LTA test results with ADP as an aggregation inducer did not show any statistical significance when compared to clopidogrel users and non-users. The impedance aggregometry results of the ASPI test did not differ between patients treated with dual antiplatelet therapy and those treated with aspirin alone. Therefore, we can assume that clopidogrel did not affect the findings.

Our results did not show any correlation between the administration of proton pump inhibitors, higher BMI values, diabetes mellitus, and chronic kidney disease which have been reported to be risk factors for the development of HTPR on ASA treatment [23, 31-33] - and LTA or impedance aggregometry results. This is probably caused by the small number of subjects in our study.

\section{Conclusions}

The present study shows an association between the use of dipyrone and HTPR in patients with PAD. Our data suggest that a causal relationship exists between HTPR and dipyrone administration. However, this study does not allow us to draw any firm conclusion due to the small number of patients tested. Thus, prospective randomised trials are needed to confirm our findings. The importance of this finding is stressed by the fact that treatment of postoperative pain with the use of dipyrone is common in many countries.

\section{Acknowledgements}

This clinical research project was supported by the Ministry of Health, Czech Republic - Conceptual Development of Research Organization VFN64165, Charles University Project Progress Q25, and Charles University Grant UNCE 204022. 


\section{Statement of Ethics}

This study was approved by the institutional ethics committee. Written consent was obtained from all patients.

\section{Disclosure Statement}

The authors have no conflicts of interest to disclose.

\section{References}

$\checkmark 1$ Collaborative overview of randomised trials of antiplatelet therapy - I: prevention of death, myocardial infarction, and stroke by prolonged antiplatelet therapy in various categories of patients. Antiplatelet Trialists' Collaboration. BMJ 1994;308:81-106.

$\checkmark 2$ Krasopoulos G, Brister SJ, Beattie WS, et al: Aspirin "resistance" and risk of cardiovascular morbidity: systematic review and metaanalysis. BMJ 2008;336:195-198.

$>3$ Feher G, Feher A, Pusch G, et al: Clinical importance of aspirin and clopidogrel resistance. World J Cardiol 2010;2:171-186.

$\checkmark 4$ Paniccia R, Priora R, Liotta AA, et al: Platelet function tests: a comparative review. Vasc Health Risk Manag 2015;11:133-148.

5 Mueller MR, Salat A, Stangl P, et al: Variable platelet response to low-dose ASA and the risk of limb deterioration in patients submitted to peripheral arterial angioplasty. Thromb Haemost 1997;78:1003-1007.

6 Pusch G, Feher G, Kotai K, et al: Aspirin resistance: focus on clinical endpoints. J Cardiovasc Pharmacol 2008;52:475-484.

$>7$ Snoep JD, Hovens MM, Eikenboom JC, et al: Association of laboratory-defined aspirin resistance with a higher risk of recurrent cardiovascular events: a systematic review and meta-analysis. Arch Intern Med 2007;167:15931599.

8 Marcucci R, Paniccia R, Antonucci E, et al: Usefulness of aspirin resistance after percutaneous coronary intervention for acute myocardial infarction in predicting one-year major adverse coronary events. Am J Cardiol 2006;98:1156-1159.

$>9$ Gianetti J, Parri MS, Sbrana S, et al: Platelet activation predicts recurrent ischemic events after percutaneous coronary angioplasty: a 6 months prospective study. Thromb Res 2006; 118:487-493.

10 Papp J, Sandor B, Vamos Z, et al: Antiplatelet effect of acetylsalicylic acid, metamizole and their combination - in vitro and in vivo comparisons. Clin Hemorheol Microcirc 2014; 56 $1-12$.

$>11$ Saxena A, Balaramnavar VM, Hohlfeld T, et al: Drug/drug interaction of common NSAIDs with antiplatelet effect of aspirin in human platelets. Eur J Pharmacol 2013;721:215-224.
12 Kurth T, Glynn RJ, Walker AM, et al: Inhibition of clinical benefits of aspirin on first myocardial infarction by nonsteroidal antiinflammatory drugs. Circulation 2003;108:11911195.

13 MacDonald TM, Wei L: Effect of ibuprofen on cardioprotective effect of aspirin. Lancet 2003;361:573-574.

$\checkmark 14$ Polzin A, Zeus T, Schrör K, et al: Dipyrone (metamizole) can nullify the antiplatelet effect of aspirin in patients with coronary artery disease. J Am Coll Cardiol 2013;62:17251726.

15 Guidelines on platelet function testing. The British Society for Haematology BCSH Haemostasis and Thrombosis Task Force. J Clin Pathol 1988;41:1322-1330.

16 Tóth O, Calatzis A, Penz S, et al: Multiple electrode aggregometry: a new device to measure platelet aggregation in whole blood. Thromb Haemost 2006;96:781-788.

17 Achilles A, Mohring A, Dannenberg L, et al: Analgesic medication with dipyrone in patients with coronary artery disease: relation to MACCE. Int J Cardiol 2017;236:76-81.

18 Gasparovic H, Petricevic M, Kopjar T, et al: Impact of dual antiplatelet therapy on outcomes among aspirin-resistant patients following coronary artery bypass grafting. Am J Cardiol 2014;113:1660-1667.

19 Kempfert J, Anger K, Rastan A, et al: Postoperative development of aspirin resistance following coronary artery bypass. Eur J Clin Invest 2009;39:769-774.

20 Poulsen TS, Kristensen SR, Korsholm L, et al: Variation and importance of aspirin resistance in patients with known cardiovascular disease. Thromb Res 2007;120:477-484.

21 Zimmermann N, Wenk A, Kim U, et al: Functional and biochemical evaluation of platelet aspirin resistance after coronary artery bypass surgery. Circulation 2003;108:542-547.

22 Petricevic M, Kopjar T, Gasparovic H, et al: Impact of aspirin resistance on outcomes among patients following coronary artery bypass grafting: exploratory analysis from randomized controlled trial (NCT01159639). J Thromb Thrombolysis 2015;39:522-531.
23 Neubauer H, Kaiser AF, Endres HG, et al: Tailored antiplatelet therapy can overcome clopidogrel and aspirin resistance - the BOchum CLopidogrel and Aspirin Plan (BOCLAPlan) to improve antiplatelet therapy. BMC Med 2011;9:3.

24 Dannenberg L, Erschoff V, Bönner F, et al: Dipyrone comedication in aspirin treated stroke patients impairs outcome. Vascul Pharmacol 2016;87:66-69.

25 Blois SL, Lang ST, Wood RD, et al: Biologic variability and correlation of platelet function testing in healthy dogs. Vet Clin Pathol 2015; 44:503-510.

26 Chakroun T, Addad F, Abderazek F, et al: Screening for aspirin resistance in stable coronary artery patients by three different tests. Thromb Res 2007;121:413-418.

27 Hohlfeld T, Zimmermann N, Weber AA, et al: Pyrazolinone analgesics prevent the antiplatelet effect of aspirin and preserve human platelet thromboxane synthesis. J Thromb Haemost 2008;6:166-173.

28 Polzin A, Richter S, Schrör K, et al: Prevention of dipyrone (metamizole) induced inhibition of aspirin antiplatelet effects. Thromb Haemost 2015;114:87-95.

29 Tuzuner Oncul AM, Yazicioglu D, Alanoglu $\mathrm{Z}$, et al: Postoperative analgesia in impacted third molar surgery: the role of preoperative diclofenac sodium, paracetamol and lornoxicam. Med Princ Pract 2011;20:470-476.

30 Lakha SF, Pennefather P, Badr HE, et al: Health services for management of chronic non-cancer pain in Kuwait: a case study review. Med Princ Pract 2016;25(suppl 1):2942.

31 Henry P, Vermillet A, Boval B, et al: 24-hour time-dependent aspirin efficacy in patients with stable coronary artery disease. Thromb Haemost 2011;105:336-344.

32 Würtz M, Grove EL, Kristensen SD, et al: The antiplatelet effect of aspirin is reduced by proton pump inhibitors in patients with coronary artery disease. Heart 2010;96:368-371.

33 Polzin A, Dannenberg L, Sansone R, et al: Antiplatelet effects of aspirin in chronic kidney disease patients. J Thromb Haemost 2016;14: 375-380. 